\title{
Effects of a Deeper Countermovement on Vertical Jump Biomechanics after Three Weeks of Familiarisation - Preliminary Findings
}

\author{
Wendy Balster ${ }^{1,2}$, Cheryl Xue Er Lim¹, Pui Wah Kong ${ }^{1, *}$ \\ ${ }^{1}$ Physical Education and Sports Science Academic Group, National Institute of Education, Nanyang Technological University, Singapore \\ ${ }^{2}$ Department of Biomedical Engineering, University of Twente, The Netherlands
}

Copyright $\bigcirc 2016$ by authors, all rights reserved. Authors agree that this article remains permanently open access under the terms of the Creative Commons Attribution License 4.0 International License

\begin{abstract}
This pilot study investigated whether a deeper countermovement was beneficial after a training period for neuromuscular adaptation. Nine male participants practised deep countermovement jumps $(2$ sets $\times 10$ jumps $)$ at home daily for three weeks. After training, the kinematics and ground reaction forces of normal and deep countermovement jumps were assessed. Adopting a deeper countermovement resulted in lower squat depth [normal: 0.38 (0.08) m, deep: $0.50(0.09) \mathrm{m}, p=.008]$ and greater range of motion at the ankle, knee and hip (all $p<.05$ ). Participants did not, however, improve in jump height [normal: $0.58(0.08) \mathrm{m}$, deep: $0.59(0.09) \mathrm{m}, p=.314]$. Squatting down deeper does not have necessary result in a higher jump height for all individuals. The lack of difference in jump height may be explained by insufficient training duration and intensity, and/or poor use of arm swing in the deep countermovement jumps. Future studies can confirm these preliminary results with more participants.
\end{abstract}

Keywords Neuromuscular Adaptation, Training, Impulse, Kinematics, Ground Reaction Force

\section{Introduction}

The influence of starting position and squat depth on maximum vertical jump performance has received considerable attention recently [e.g.1-5]. For standing jumps, the vertical jump height achieved in flight is determined by the impulse generated during ground contact. Adopting a deeper squat position allows a longer time for muscles to generate force [7]. This will increase the impulse and therefore enhance jump performance, given that at least the same amount of force is produced.

Simulation studies have investigated the influence of initial jump positions, mainly characterised by the degree of flexion at the hips and the knees, on vertical jump height [6-9]. In squat jumps, a 4-segment model was used to show that higher jump height can be achieved from a deeper starting position [7, 9]. It has once been proposed that the same muscle activation pattern can be used regardless of the initial jump position to achieve good jump heights [9]. Bobbert et al. [6] later concluded from their simulations with experimental electromyographic inputs that changes in the initial squat posture required adjustments in muscle stimulation patterns to achieve maximum jump height. For countermovement jumps, Selbie and Caldwell [8] found that maximal jump height was relatively insensitive to initial posture, given that the pattern of joint torque onset times were optimised to allow the body to re-orient itself during the downward countermovement phase. Collectively, these theoretical studies suggest that with optimised muscle activation patterns, the human body can achieve improved jump heights from deep squat positions.

Findings from experimental studies on human participants, however, are less clear regarding the potential benefits of adopting a deeper squat position $[4-7,10,11]$. Domire and Challis [7] compared the squat jump heights between preferred and deep squat depths in 10 male participants and found no difference in performance. Among participants who are proficient jumpers, there was a trend that increasing squat depth corresponded to an increase in jump height for both squat and countermovement jumps [4-6, 10, 11]. While higher jump heights were usually observed between the very shallow and the deepest squat depths (e.g. $15 \mathrm{~cm} 75 \mathrm{~cm}$ [10]), no further improvements in squat or countermovement jumps could be seen by adopting deeper than the preferred squat depth [4-6, 10].

It should be noted that all previous experimental studies asked participants to jump from various squat positions on one occasion without much prior practice and therefore it is unlikely that these jumps were optimally coordinated [6,7]. The limitation of participants being unable to optimally 
activate their leg muscles from a deep squat position due to inexperience can be addressed by including a training period to allow for neuromuscular adaptation. Thus, the purpose of the present study was to investigate whether a deeper countermovement could improve vertical jump performance after a training period for neuromuscular adaptation. It was hypothesized that after training a higher jump height could be achieved by adopting a deeper countermovement when compared to a normal jump. Given the constraints in resources, only a small scale pilot study was conducted. The preliminary results obtained may guide future studies and provide important information for athletes, coaches, physical education teachers and exercise professionals to develop training protocols for enhancing jump performance

\section{Materials and Methods}

\subsection{Experimental Approach}

This study used a within-subject randomised cross-over design to approach the research problem. Following three weeks of jump training, subjects performed normal and deep countermovement vertical jumps (three trials each, order randomised) in the Sports Biomechanics Laboratory. Jumping performances were recorded using a force platform and a three-dimensional motion capture system. The biomechanics of the best normal and best deep jumps were compared.

\subsection{Participants}

This study was ethically approved by the Nanyang Technological University Institutional Review Board was performed in accordance with the Helsinki Declaration. All participants were informed of any risks associated with participation in the study and were free to withdraw if they desired. Written informed consent was obtained from all subjects prior to any experimental procedures. Nine recreationally active male university students [mean $(\mathrm{SD})$ : age $=21.9(2.0)$ yrs., mass $=71.3(11.9) \mathrm{kg}$, height $=174.0$ $(5.2) \mathrm{cm}]$ were recruited using convenient sampling. Participants must have exercised at least 3 times per week in the past three months prior to the start of the study to be eligible. Those who had sustained an injury in the three months before the start of the study were excluded.

\subsection{Procedures}

The body mass and height of eligible participants were taken. All participants attended a familiarisation session in which the procedures were identical to the main test trial (to be described later). At the end of the familiarisation session, participants were taught a training drill to promote neuromuscular adaptation to a deeper countermovement (Figure 1). To ensure that the participants adopted a sufficiently deep squat position, they had to touch the ground with both hands before jumping up. Participants were then asked to practise this drill ( 2 sets of 10 jumps) daily for three weeks in their own time before returning to the laboratory for the main test. This training volume corresponded to 21 sessions or 420 jumps, which was within the range (2-3 times a week for 6-15 weeks) needed for enhancement of jumping performance [12]. Participants were provided with a training $\log$ to record their daily training routine and to report other issues such as injuries. A minimum of 14 out of 21 sessions (or two-third) was set as the compliance criteria. On average, participants completed 18.3 (SD 2.7, range 14 to 21) training sessions and returned to the laboratory for the main test in 22.7 (SD 4.7, range 20 to 35 ) days.

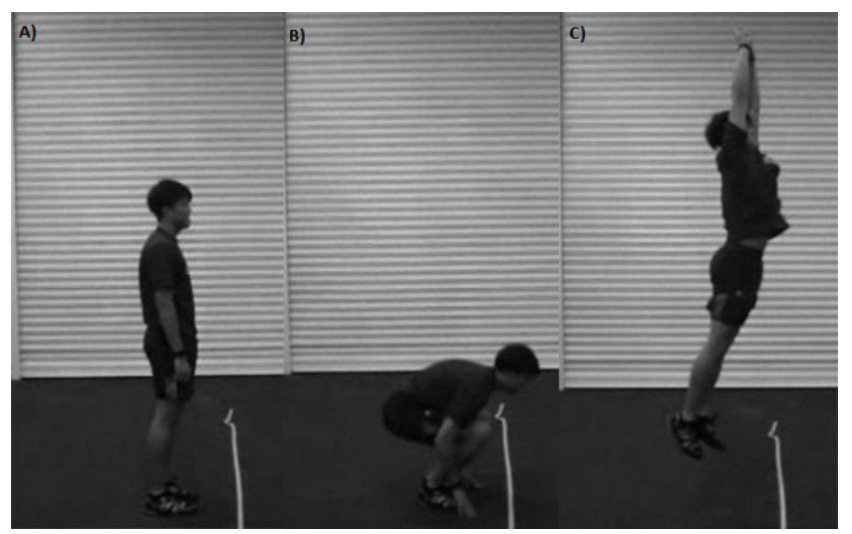

Figure 1. Participants were instructed to perform 2 sets of 10 deep vertical countermovement jumps daily for three weeks: A) starting in an upright position, B) adopting a deep countermovement with both hands touching the ground, and C) jumping up vertically at maximal effort.

On the main test day, participants warmed up for jump tests using their own routine. Each participant performed six maximal effort vertical jumps - three with a normal and three with a deep countermovement. The jump orders for the countermovement conditions were randomized, with a two-minute rest interval between successive jumps. No restrictions in the arm movements were imposed so as to replicate realistic situations in most sports. Participants were asked to swing their arms naturally without having to touch the ground for the deep jumps. To collect three-dimensional kinematic data, 34 retroreflective markers were placed on the participants prior to the jump trials (Figure 2). All jump performances were recorded by an 8-camera motion capture system via the Cortex software (Motion Analysis Corporation, Santa Rosa, CA, USA) at $200 \mathrm{~Hz}$. Ground reaction forces (GRF) were sampled in synchronisation with the motion data using a force platform (AMTI, Watertown, MA, USA) at $2000 \mathrm{~Hz}$.

Data were processed using the Visual3D software (C-Motion, Inc., Rockville, MD, USA). Vertical GRF of all jumping trials were low-pass filtered at $50 \mathrm{~Hz}$ and normalized to individual participants' body weights (BW). Takeoff was defined as the first frame where the vertical GRF fell below $0.001 \mathrm{BW}$. The marker coordinates were low pass filtered at $8 \mathrm{~Hz}[6,13]$. From the 34 marker positions, a 
13-segment model was constructed (Figures 2 and 3). The centre of mass (CM) vertical position was identified at: 1) static quiet standing in a T-position, 2) lowest position during countermovement, 3) takeoff, and 4) highest position in flight (Figure 3). Subsequently, the squat depth (CM standing - $\mathrm{CM}$ lowest), $\mathrm{CM}$ height raised at takeoff (CM takeoff - CM standing), CM vertical displacement in flight (CM highest - CM takeoff) and total jump height (CM highest - CM standing) for each trial were calculated. Out of the three trials per countermovement condition, the jump with the highest total jump height was selected for further kinematic and kinetic analysis [14-16].
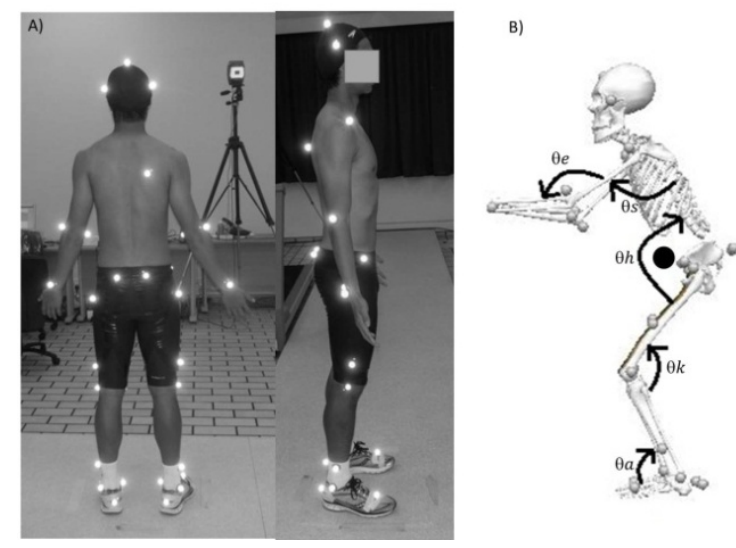

Figure 2. A) 34 retroreflective markers, $B$ ) centre of mass and joint angle definitions $\left(\theta_{\mathrm{a}}=\right.$ ankle angle, $\theta_{\mathrm{k}}=$ knee angle, $\theta_{\mathrm{h}}=$ hip angle, $\theta_{\mathrm{s}}=$ shoulder angle, $\theta_{\mathrm{e}}=$ elbow angle). The arrows indicate positive direction of each joint.

For the best normal and best deep jump trials, push-off time was determined as the upward duration from the lowest $\mathrm{CM}$ position to takeoff [13]. Joint angles and angular velocities of the ankle, knee, hip, shoulder and elbow were calculated (Figure 2B). The range of motion (ROM) of each joint during the upward jump duration (from CM lowest to takeoff) was calculated. The average ROM of the left and right sides were used for statistical analysis. The peak vertical force and net vertical jump impulse (from CM lowest to takeoff) were determined.

\subsection{Statistical Analysis}

Twelve dependent variables were chosen for statistical analysis: squat depth, $\mathrm{CM}$ height raised at takeoff, $\mathrm{CM}$ vertical displacement in flight, total jump height, push-off time, peak GRF, net vertical jump impulse, and ROM of the ankle, hip, knee, shoulder and elbow. Descriptive statistics were calculated and expressed as group means (SD). For each variable of interest, the Wilcoxon signed rank-test was performed (SPSS version 21.0, Chicago, IL) to detect differences between the normal and deep countermovement vertical jumps. Statistical significance was set at $P<.05$. Effect size $(r)$ was calculated to describe the magnitude of the difference and interpreted as follows: small 0.1 to 0.29 , medium 0.3 to 0.49 , large $\geq 0.5$.

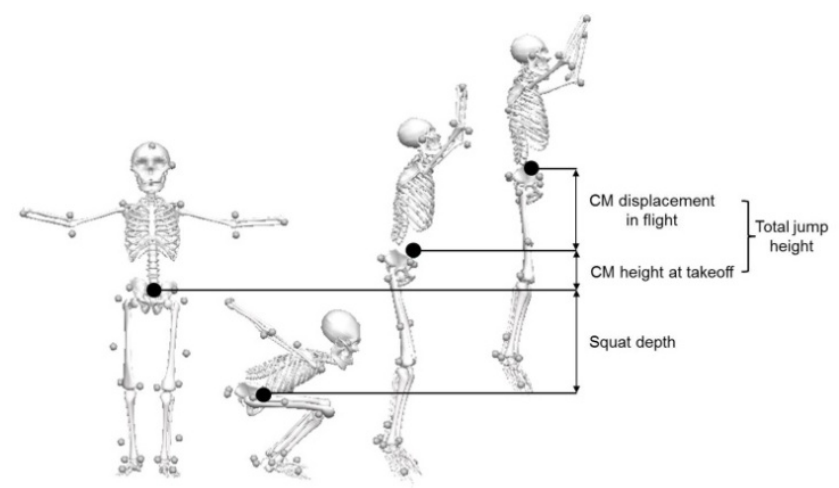

Figure 3. The centre of mass (CM) position was used to determine squat depth, $\mathrm{CM}$ height raised at takeoff, $\mathrm{CM}$ vertical displacement in flight and total jump height.

\section{Results}

As a group, the mean squat depth was lowered by $12 \mathrm{~cm}$ $(P=.008)$ in the deep countermovement jump but this did not result in a significant increase in total jump height $(1 \mathrm{~cm}$, $P=.314$, Table 1). Large inter-individual variations were observed, with six participants jumping higher $(+0.7$ to +6.8 $\mathrm{cm})$ and three lower $(-0.1$ to $-3.2 \mathrm{~cm})$ from a deeper countermovement (Figure 4A). The difference in total jump height was within $5 \%$ regardless of the countermovement depth for all except one participant (deep $>$ normal by 12.1\%). No trend between training compliance and difference in total jump height was seen (Figure 4B).

There was a $20.0 \%$ increase in push-off duration for the deep countermovement condition but the peak GRF was $6.7 \%$ lower (Table 1). As shown in Figure 5, there were large variations in the GRF profiles, with some trials displaying a single peak and others a double peak pattern.

Overall the joint kinematic patterns were similar between the two jumps, with more variation observed at the shoulder (Figures 6 and 7). Adopting a deeper countermovement resulted in an increase in ROM at the ankle, knee and hip compared to the normal jump (Table 1, Figure 6). No kinematic differences were observed at the elbow or shoulder joints. Interestingly, one participant swung his arms downwards during the push-off phase of his deep jump, as opposed to the normal upward arm swing (Figure 6). 
Table 1. Comparison of kinematic and kinetic variables between normal and deep countermovement vertical jumps

\begin{tabular}{|c|c|c|c|c|}
\hline Variable & Normal Jump & Deep Jump & $P$-value & Effect Size $(r)$ \\
\hline Squat depth (m) & $0.38(0.08)$ & $0.50(0.09)$ & $.008 *$ & -0.628 \\
\hline CM height at takeoff (m) & $0.12(0.02)$ & $0.12(0.02)$ & .066 & -0.434 \\
\hline CM displacement in flight (m) & $0.46(0.07)$ & $0.47(0.08)$ & .477 & -0.168 \\
\hline Total jump height (m) & $0.58(0.08)$ & $0.59(0.09)$ & .314 & -0.237 \\
\hline Push-off time (s) & $0.30(0.04)$ & $0.36(0.05)$ & $.008^{*}$ & -0.629 \\
\hline Peak force (BW) & $2.52(0.20)$ & $2.35(0.22)$ & $.038^{*}$ & -0.489 \\
\hline Net vertical impulse (BW.s) & $0.30(0.03)$ & $0.30(0.03)$ & .214 & -0.293 \\
\hline $\operatorname{ROM} \theta_{\mathrm{a}}\left(^{\circ}\right)$ & $54(7)$ & $60(7)$ & $.011^{*}$ & -0.600 \\
\hline $\operatorname{ROM} \theta_{\mathrm{k}}\left({ }^{\circ}\right)$ & $85(15)$ & $108(19)$ & $.008^{*}$ & -0.629 \\
\hline $\mathrm{ROM} \theta_{\mathrm{h}}\left(^{\circ}\right)$ & $67(12)$ & $76(9)$ & $.008^{*}$ & -0.628 \\
\hline $\operatorname{ROM} \theta_{\mathrm{s}}\left(^{\circ}\right)$ & $138(42)$ & $135(86)$ & .859 & -0.042 \\
\hline $\operatorname{ROM} \theta_{\mathrm{e}}\left({ }^{\circ}\right)$ & $-31(25)$ & $-32(31)$ & .859 & -0.042 \\
\hline
\end{tabular}

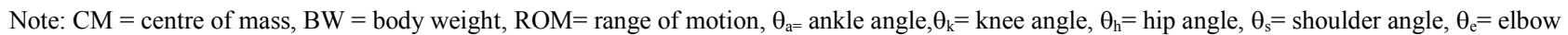
angle. *Significant difference $(P<.05)$ detected by Wilcoxon signed rank-test.

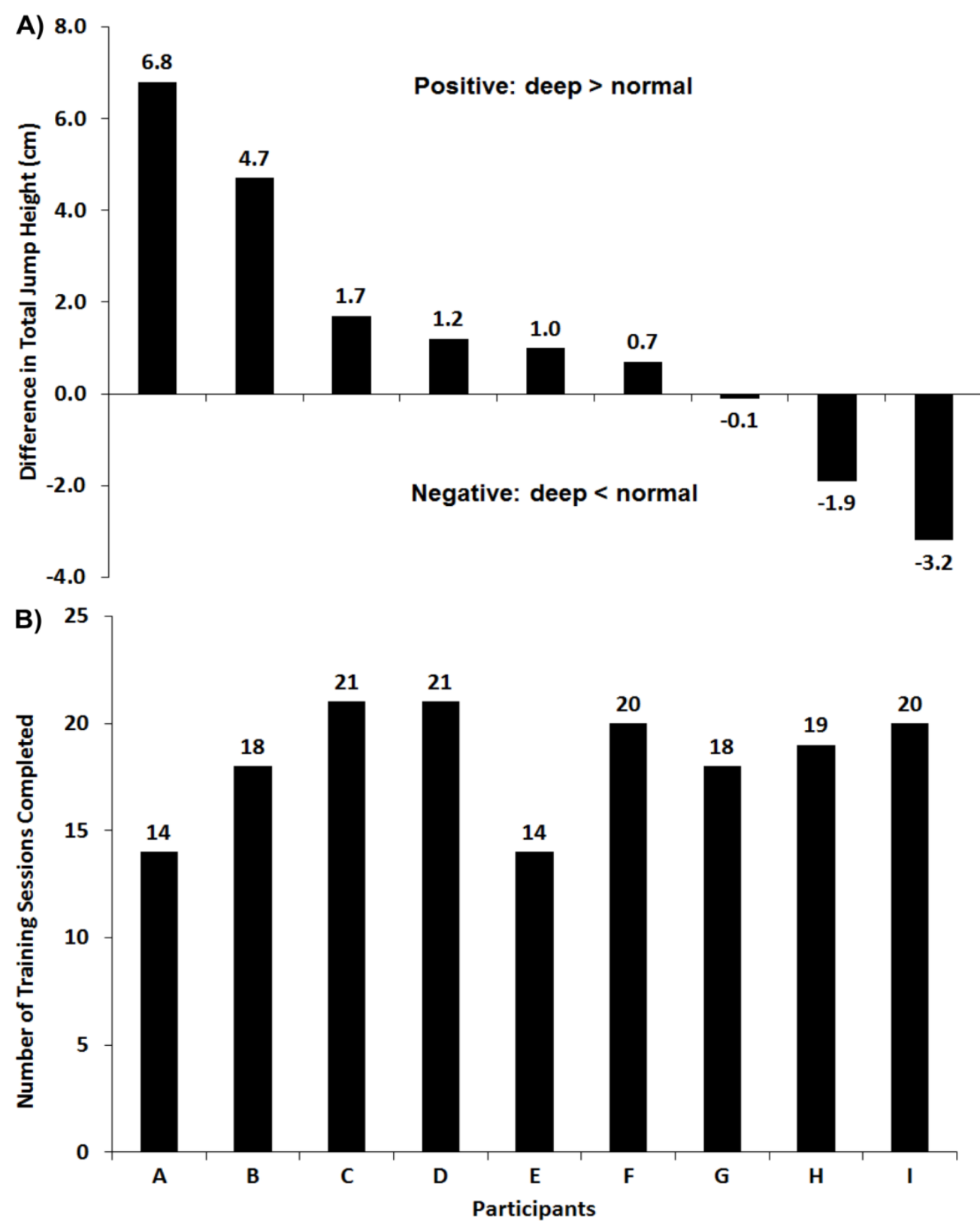

Figure 4. Individual participant's data of A) difference in total jump height (deep - normal) between deep and normal countermovement conditions, and B) number of training sessions completed. 

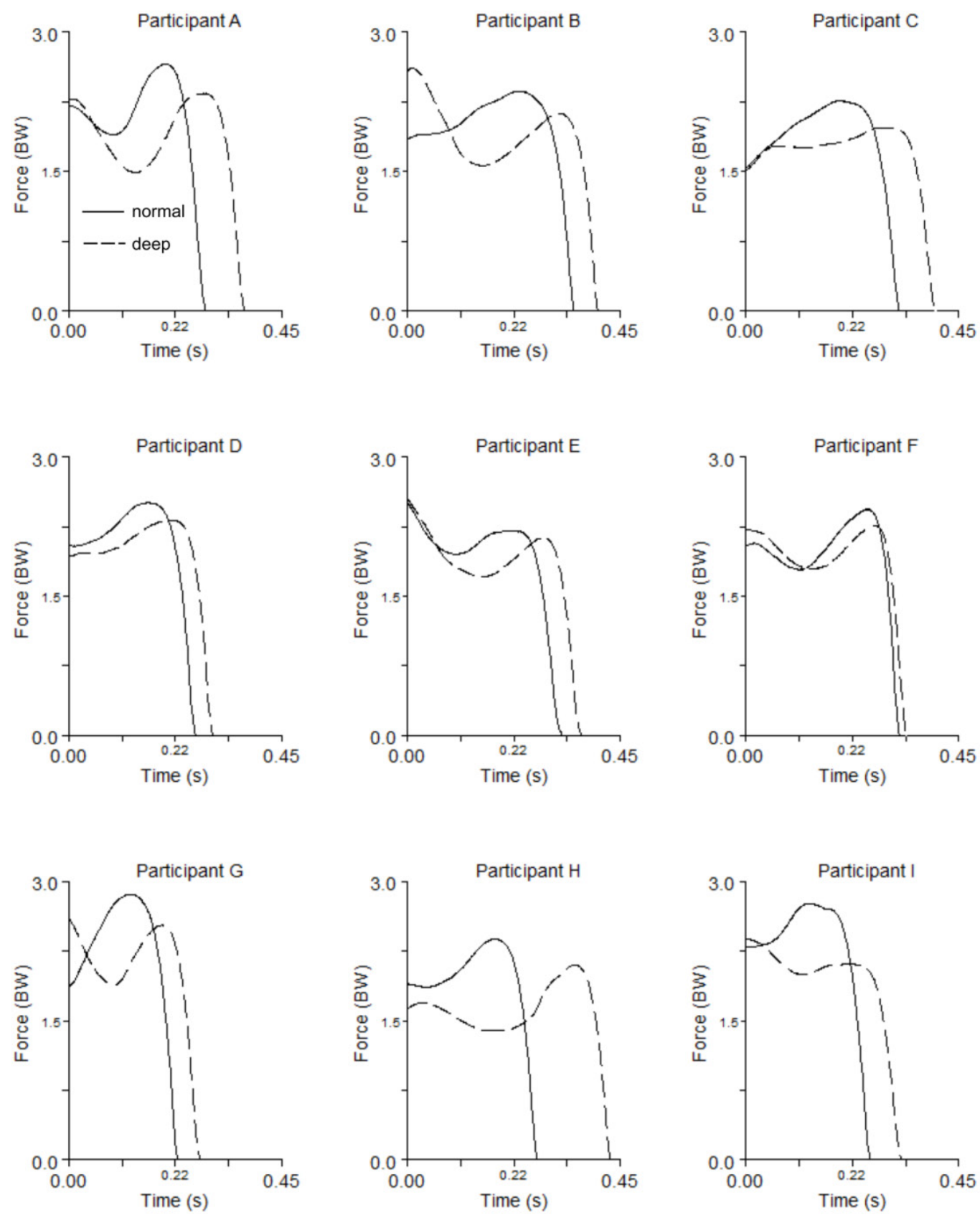

Figure 5. Vertical ground reaction forces of individual participants during the push-off phase of normal and deep vertical jumps (BW = body weight). 

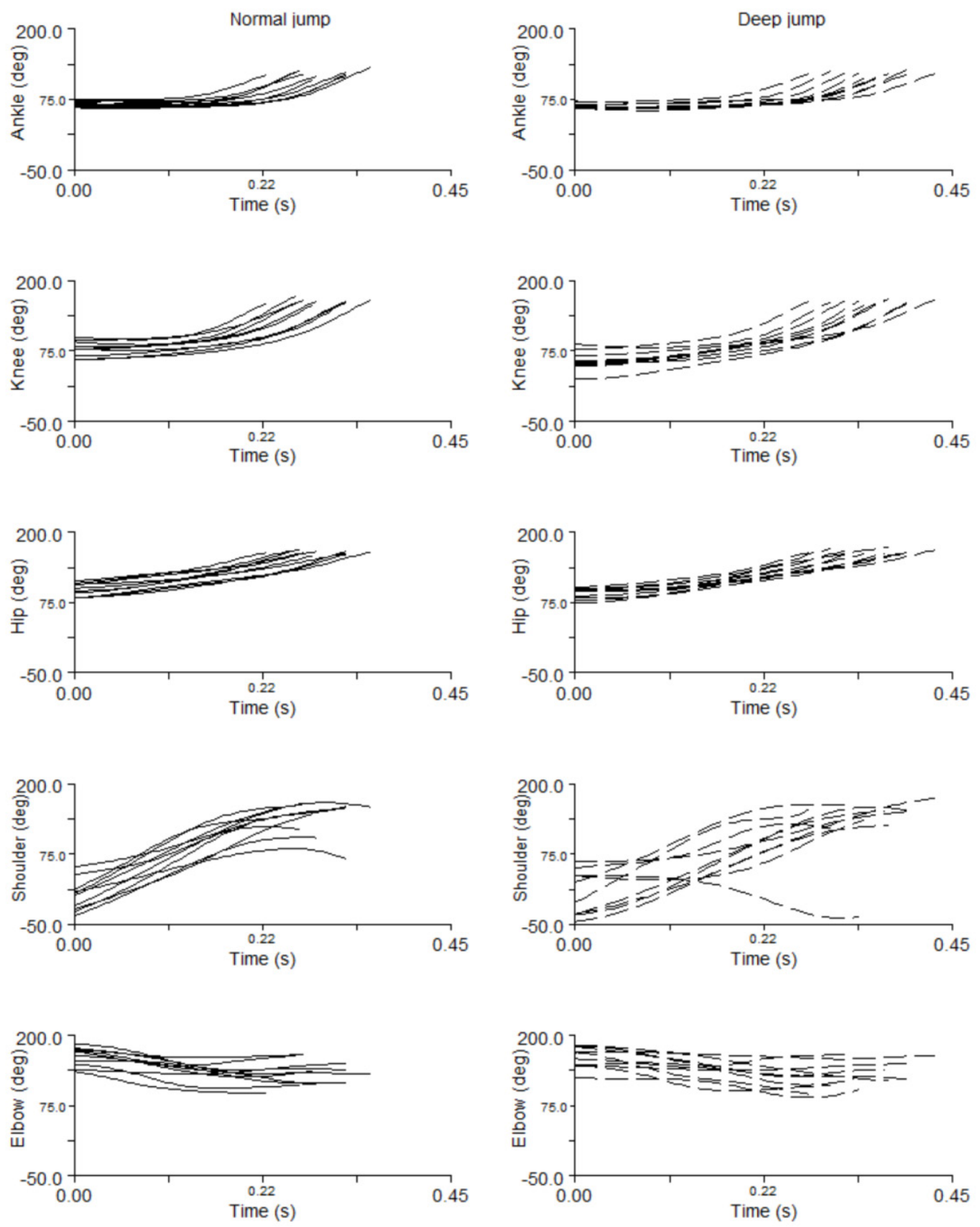

Figure 6. Joint angles of individual participants during the push-off phase of normal and deep vertical countermovement jumps. 

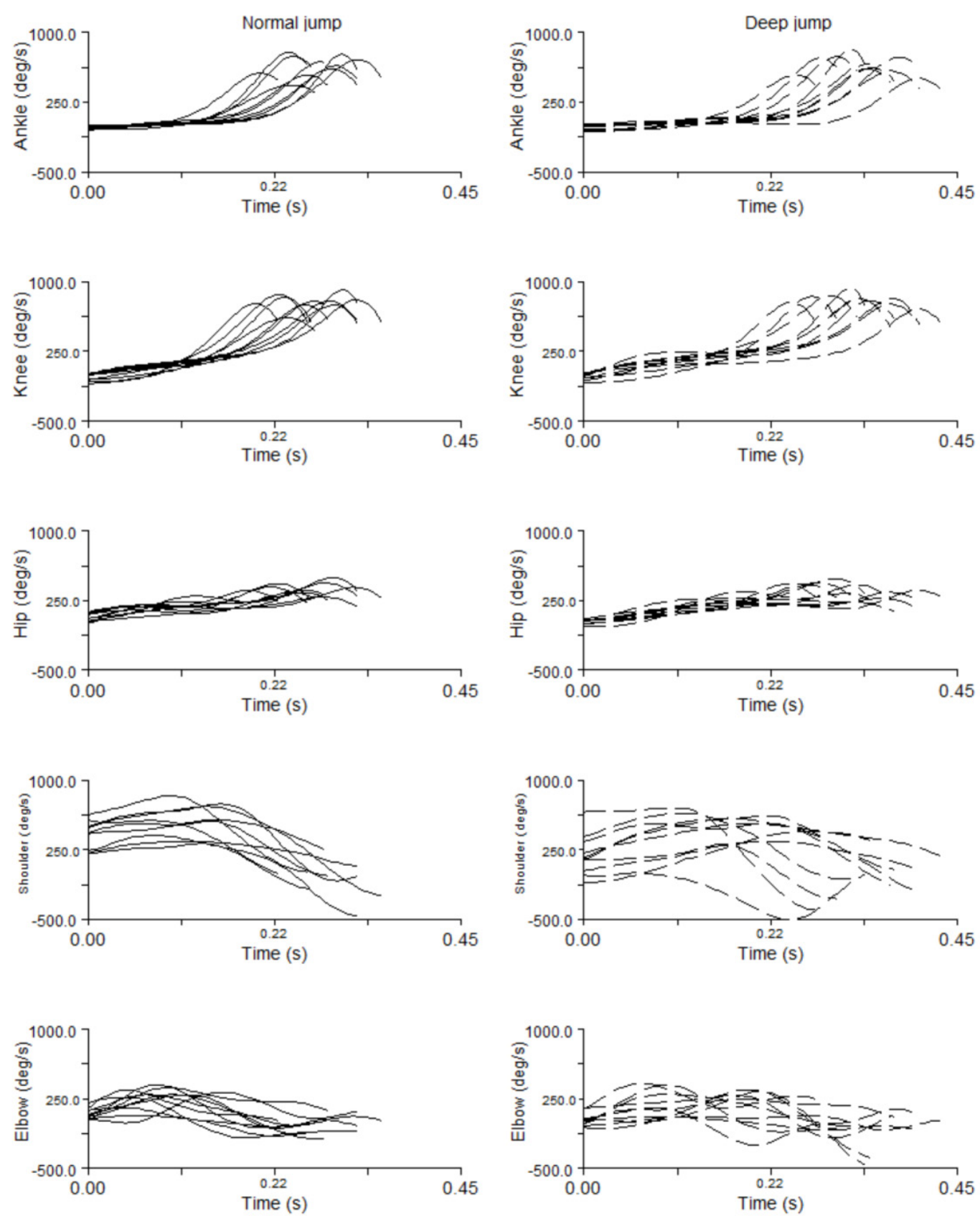

Figure 7. Joint angular velocities of individual participants during the push-off phase of normal and deep verticalcountermovement jumps.

\section{Discussion}

The purpose of this pilot study was to compare the vertical jump heights when employing normal and deep countermovements, after a training phase to allow for neuromuscular adaptation. In contrast to our hypothesis, a deeper countermovement did not result in a higher total jump height even after three weeks of familiarisation to the deep jumps.

Our results of similar jump heights between preferred and deeper countermovements parallel with many previous experimental findings with no training period $[6,7,10,77]$. The lack of differences observed, despite having three weeks of familiarisation training, may be attributed to a number of reasons as explained below.

\subsection{Training}

Despite a $20.0 \%$ increase in the time available to generate force, participants were unable to increase the jump impulse due to the decrease in force production (Table 1, Figure 5). The deeper squat position requires muscles to operate beyond the region of optimal length where the capability for force production is low. Since motor unit activation varies with joint angles [17,18], a three-week training protocol designed for improving neuromuscular coordination when jumping from an unaccustomed deep squat position was implemented. At the pilot stage of this study, we have 
collected kinematic data on 5 participants before and after three weeks of deep jump training [19]. Participants jumped approximately $3 \mathrm{~cm}$ higher in both normal and deep jumps after the training, though the improvement of adopting a deeper countermovement appeared similar between the pre(deep $=2.7 \mathrm{~cm}$ higher) and post-tests (deep $=2.5 \mathrm{~cm}$ higher). These data suggest some training effect in our protocol but perhaps not sufficient to demonstrate the potential of jumping from a deep position. One previous study showed that six weeks of twice-weekly plyometric drop-jumping from $50 \mathrm{~cm}$ with a total of 140 jumps resulted in significantly increased vertical jump height [14]. In comparison, our participants had performed more jumps (mean $=327$, range 140 to 420 ) though the training intensity was much lighter. Another study found improvement in jump height after 16 weeks of heavy resistance and explosive exercises [20]. Thus, it is possible that our training intensity and/or duration were not sufficient to facilitate muscle activation coordination.

On the other hand, neuromuscular coordination may not be the key determining factor of improving jump height from a deep position. Perhaps greater muscular strength is needed before one can benefit from a deep countermovement jump as there is likely an increased in initial strain on the joints at the deep position [21]. This speculation also aligns with the observations that a deep countermovement technique was sometimes seen among platform divers and volleyball players competing at the elite level (e.g. Olympic Games). Participants in this study, though recreationally active, may not have been able to overcome the greater range of motion in the deep jump condition (see Table 1) because of their low leg strength. The training period in this study did not intend to induce muscle hypertrophy or increase muscular strength. This would require a longer duration, higher training volume and intensity [22].

With sufficient training, it is evident that length-specific strength can be developed in human skeletal muscles as seen in elite athletes competing in different sports [23]. Recently, an interesting study by Ullrich and colleagues [24] demonstrated that squat jumping mechanics are altered after length-specific muscle training. In their study, 16 athletes underwent eight weeks of strength training regimens that were restricted to long muscle-tendon unit length for the knee extensors and flexors. After training, squat jump height improved and the isometric moment-knee angle relationship of the knee extensors was shifted to longer muscle-tendon unit lengths. Thus, future studies can consider incorporating a longer training protocol to improve not only neuromuscular coordination but also lower extremity muscular strength. Comparing samples with different levels of leg strength may also help clarify whether only well-trained individuals with greater lower extremity strength can benefit from adopting a deeper-than-preferred countermovement in vertical jumps.

\subsection{Influence of Armswing}

Effective use of an armswing can enhance vertical jump height substantially [13, 25-27]. Although there was no difference as a group, we observed reduced shoulder ROM for the deep countermovement jumps in four out of nine participants, including one swinging the arm in the wrong direction. The reduced ROM may reflect a less effective armswing, compromising on the work done by the shoulder and the hip joints to increase jump height [26, 28, 29]. An ineffective use of armswing may have also masked the effect of any improved muscular work from the lower extremities in the deep jumps. It was once proposed that the GRF in the latter half of the push-off phase was increased by the use of the arms [27]. Another study failed to identify clear relationships between force-time pattern and the use of armswing, with some subjects showing single GRF peak and others double peaks despite all using an armswing [30]. In our study, a mixture of single/double peak in GRF was observed (Figure 5). Most normal jumps peaked during the latter phase of the push-off while the deep jumps displayed more variations in the timings of the peaks. The more variable GRF pattern in the deep jumps may be partly explained by the different armswing kinematics (Figures 6 and 7). Future studies can further clarify the inter-relationship among armswing, countermovement depth and GRF patterns.

During the jumping tests in this study, arm movement was not restricted so as to simulate a more realistic scenario such as in sports contexts. Keeping the hands on the hips can certainly eliminate the inconsistent armswing patterns in the participants and therefore allow a clearer comparison of the lower extremity mechanics between the deep and normal countermovements. However, this approach is impractical as athletes would normally swing their arms during jumping. An alternative solution may to be design a training drill that also emphasizes effective use of the armswing during a deep countermovement jump. In this way, participants can optimize the muscle activation pattern in a realistic way as they would use in a sports setting.

\subsection{Inter-individual Differences}

The large inter-individual variations observed suggests that some participants can benefit from adopting a deeper countermovement. For example, Participant A jumped $12.1 \%$ higher in the deep position (Figure 4A) and this improvement is considered substantial in sports performance. With the data available, we failed to identify any distinguishable patterns between the responders (Participants A to F) and non-responders (Participants G, H, I). One may suspect the non-responders to be those who did not use the armswing effectively or missed many training sessions. Participant F, despite swinging his arms in the wrong direction during the deep countermovement condition, still managed to jump $1.2 \%$ higher. The poor armswing technique may have been compensated by his strong commitment to training, completing 20 out of 21 sessions but overall the benefit of a deep countermovement does not relate to 
training compliance (Figure 4B). Participants A (12.1\%) and E (1.7\%) both jumped higher when adopting a deep countermovement despite performing only 14 out of the 21 sessions. Thus, there is a need to better understand the underlying factors that caused the large inter-individual variations in the response to a deeper-than-preferred countermovement.

\subsection{Limitations}

There are a few limitations to this study. First, the training was unsupervised. Although a $\log$ book was provided to remind participants to perform the jumping drills every day, some missed several sessions and therefore may not have sufficiently adapted to jumping from a deeper position. Second, we instructed participants to touch the ground with both hands to ensure that they squatted down low enough during their training at home. However, participants could have achieved this solely through greater hip flexion by leaning forward, thereby compromising on the knee and ankle flexions. Reaching down to touch the floor may have also discouraged the arms from swinging backward, thus leading to an ineffective armswing during the deep jumps. Finally, our sample size was relatively small with diverse backgrounds in physical activity participation. Inter-individual differences in the response to a deeper countermovement may be reduced for a more homogenous sample. Future studies with larger sample size are needed to confirm the preliminary results reported in this pilot study.

\section{Conclusions}

The present study showed that after three weeks of training for neuromuscular coordination, vertical jump height did not improve by adopting a deeper-than-preferred countermovement in recreationally active participants. Large individual variations were observed with participants jumping up to $12.1 \%$ higher from a deep position. While such an improvement may be substantial for sports performance, coaches and athletes should be aware that squatting down deeper does not necessary result in a higher jump height for all individuals. Future studies can include more participants and consider designing a training protocol to allow for muscular hypertrophy alongside neuromuscular adaptation. If feasible, trainings should be supervised to ensure completion and proper countermovement and armswing techniques. There is also a need to better understand the large inter-individual variations in the response to a deeper than preferred countermovement.

\section{Acknowledgements}

We wish to acknowledge the funding support for this project from Nanyang Technological University under the
Undergraduate Research Experience on Campus (URECA) programme. Our thanks also go to Dr. Qu Xingda and Mr. Edwin Lam for their assistance in data collection. The authors declare that there is no conflict of interest.

\section{REFERENCES}

[1] M. F. Bobbert, L. J. Casius. Is the effect of countermovement on jump height due to active state development? Medicine and Science in Sports and Exercise, Vol. 37, 440-446, 2005.

[2] Y. Blache Y, K. Monteil. Effects of spine flexion and erector spinae maximal force on vertical squat jump height: a computational simulation study. Sports Biomechanics, Vol. 14, No. 1, 81-94, 2015.

[3] Z. J. Domire, J. H. Challis. Maximum height and minimum time vertical jumping. Journal of Biomechanics, Vol. 48, No. $11,2865-2870$

[4] R.G. Gheller, J. Dap Pupo, J. Ache-Dias, D. Detanico, J. Padulo, S. G. dos Santos. Effect of different knee starting angles on intersegmental coordination and performance in vertical jumps. Human Movement Science, Vol 42, 71-80.

[5] R.G. Gheller, J. Dap Pupo, L. A. P. de Lima, B.M. de Moura, S. G. dos Santos. Effect of squat depth on performance and biomechanical parameters of countermovement vertical jumps. Revista Brasileira de Cineantropometria e Desempenho Humano, Vol. 16, No. 6, 658-668, 2014.

[6] M. F. Bobbert, L. J. Casius, I. W. T. Sijpkens, T. J. Jaspers. Humans adjust control to initial squat depth in vertical squat jumping. Journal of Applied Physiology, Vol. 105, 1428-1440, 2008.

[7] Z. J. Domire, J. H. Challis. The influence of squat depth on maximal vertical jump performance. Journal of Sports Sciences, Vol. 25, 193-200, 2007.

[8] W. S. Selbie, G. E. Caldwell. A simulation study of vertical jumping from different starting postures. Journal of Biomechanics, Vol. 29, 1137-1146, 1996.

[9] A.J. van Soest, M. F. Bobbert, G. J. van Ingen Schenau. A control strategy for the execution of explosive movements from varying starting positions. Journal of Neurophysiology, Vol. 71, 1390-1402, 1994.

[10] T.J. Kirby, J. M. McBride, T. L. Haines, A. M. Dayne. Relative net vertical impulse determines jumping performance. Journal of Applied Biomechanics, Vol. 27, 207-214, 2011.

[11] J.M. McBride, T.J. Kirby, T.L. Haines, J. Skinner. Relationship between relative net vertical impulse and jump height in jump squats performed to various squat depths and with various load. International Journal of Sports Physiology and Performance, Vol. 5, No. 4, 484-496, 2010.

[12] G. Markovic, P. Mikulic. Neuro-musculoskeletal and performance adaptations to lower-extremity plyometric training. Sports Medicine, Vol. 40, 859-895, 2010.

[13] M. Hara, A. Shibayama, D. Takeshita, D.C. Hay, S. Fukashiro. A comparison of the mechanical effect of arm swing and 
countermovement on the lower extremities in vertical jumping. Human Movement Science, Vol. 27, 636-648, 2008.

[14] L. S. McClenton, L. E. Brown, J. W. Coburn, R.D. Kersey. The effect of short-term VertiMax vs. depth jump training on vertical jump performance. Journal of Strength and Conditioning Research, Vol. 22, 321-325, 2008.

[15] J. M. Sheppard, J.B. Cronin, T.J. Gabbett, M. R. McGuigan, N. Etxebarria, R. U. Newton. Relative importance of strength, power and anthropometric measures to jump performance of elite volleyball players. Journal of Strength and Conditioning Research, Vol. 22, 758-765, 2008.

[16] M. Váczi, J. Tollár, B. Meszler, I. Juhász, I. Karsai. Short-term high intensity plyometric training program improves strength, power and agility in male soccer players. Journal of Human Kinetics, Vol. 36, 17-26, 2013

[17] N. Babault, M. Pousson, A. Michaut, J. Van Hoecke. Effect of quadriceps femoris muscle length on neural activation during isometric and concentric contractions. Journal of Applied Physiology, Vol. 94, 983-990, 2003.

[18] N. A. Maffiuletti, R. Lepers. Quadriceps femoris torque and EMG activity in seated versus supine position. Medicine and Science in Sports and Exercise, Vol. 35, 1511-1516, 2003.

[19] P.W. Kong, C.X.E. Lim, Effect of squat depth on vertical counter-movement jump height - a pilot investigation, in: Bradshaw, E.J., Burnett, A. and Hume, P.A., eds, Proceeding of the 30th Conference of the International Society of Biomechanics in Sports Volume 3, Australia Catholic University, 303-306, 2012.

[20] J. M. González-Ravé, M. Delgado, M. Vaquero, D. Juarez, R.U. Newton. Changes in vertical jump height, anthropometric characteristics, and biochemical parameters after contrast training in master athletes and physically active older people. Journal of Strength and Conditioning Research, Vol. 25, 1866-1878, 2011.

[21] K. B. Shelburne, M. G. Pandy. A dynamic model of the knee and lower limb for simulating rising movements. Computer
Methods in Biomechanics and Biomedical Engineering, Vol. 5, No. 2, 149-159, 2002.

[22] G. Markovic, P. Mikulic. Neuro-musculoskeletal and performance adaptations to lower-extremity plyometric training. Sports Medicine, Vol. 40, 859-895, 2010.

[23] W. Herzog, A. C. Guimaraes, M. G. Anton, K. A. Carter-Edman. Differences in the moment - length relations of rectus femoris muscles of speed skaters/cyclists and runners. Medicine and Science in Sports and Exercise, Vol. 23, $1289-1296,1991$.

[24] B. Ullrich, K. Heinrich, J.P. Goldmann, G. P. Brüggemann. Altered squat jumping mechanics after specific exercise, International Journal of Sports Medicine, Vol. 31, 243-250, 2010.

[25] M.E. Feltner, D. J. Fraschetti, R.J. Crisp. Upper extremity augmentation of lower extremity kinetics during countermovement vertical jumps. Journal of Sports Sciences, Vol. 17, No. 6, 449-466, 1999.

[26] M. Hara, A. Shibayama, D. Takeshita, S. Fukashiro. The effect of arm swing on lower extremities in vertical jumping. Journal of Biomechanics, Vol. 39, 2503-2511, 1996.

[27] E. A. Harman, D. J. Fraschetti, R.J. Crisp. Upper extremity augmentation of lower extremity kinetics during countermovement vertical jumping. Medicine and Science in Sports and Exercise, Vol. 22, 825-833, 1999.

[28] K.B. Cheng, C. Wang, H. Chen, C. Wu, Y. Chui. The mechanisms that enable arm motion to enhance vertical jump performance - a simulation study. Journal of Biomechanics, Vol. 41, 1847-1854, 2008.

[29] Z. J. Domire, J. H. Challis. An induced energy analysis to determine the mechanism for performance enhancement as a result of arm swing during jumping. Sports Biomechanics, 9, 38-46, 2010.

[30] J.J. Dowling, L. Vamos. Identification of kinetic and temporal factors related to vertical jump performance. Journal of Applied Biomechanics, Vol. 9, 95-110, 1993. 